\title{
The Dirac Equation Is Separable On The Dyon Black Hole Metric
}

\author{
İbrahim Semiz也 \\ Department of Physics, Yale University \\ 217 Prospect St. \\ New Haven, CT 06511
}

\begin{abstract}
Using the tetrad formalism, we carry out the separation of variables for the massive complex Dirac equation in the gravitational and electromagnetic field of a four-parameter (mass, angular momentum, electric and magnetic charges) black hole.
\end{abstract}

PACS Numbers: 04.40.+c, 97.60.Lf

\footnotetext{
${ }^{1}$ e-mail(bitnet): semiz@yalehep
} 


\section{Introduction}

In studies of the behavior of a matter field on a black hole background, e.g. the possible evolution of the black hole by exchanging energy, charge and angular momentum with the field; one would like to know if the variables can be separated in the relevant field equation. Obviously, the study becomes much easier, if the system of partial differential equations can be reduced to a system of ordinary differential equations. This paper concentrates on spin- $\frac{1}{2}$ (Dirac) field.

The "no-hair" theorem in General Relativity asserts that the metrics of stationary black holes can be described uniquely by three parameters : Mass $M$, charge $Q_{e}$ (assuming the absence of magnetic charges), and angular momentum per unit mass, $a$. Therefore, when one writes a matter field equation on a black hole background, these parameters become parameters of the field equation.

The Dirac equation and its separability properties on black hole metrics have been investigated in increasingly complicated contexts. The early pioneering work was done by Brill and Wheeler [1], who separated the neutrino field equation on the Schwarzschild metric. Teukolsky [2] separated a master equation for massles spin 0, 1 and 2 and noted that the separated equations for the massless spin- $\frac{1}{2}$ have the same form, and therefore could be incorporated into the master equation. Unruh [3] showed the spin- $\frac{1}{2}$ result independently. The unexpected result that the massive Dirac Equation was also separable came from Chandrasekhar 《4. The Chandrasekhar result was generalized to the $\operatorname{Kerr}-\operatorname{Newman}(Q, a, M)$ case by Page [5] and Toop [6].

However, black holes could also have magnetic charge, if such existed. Such a black hole would acquire an additional label $Q_{m}$ for the magnetic charge. The interest in this possibility has grown since magnetic monopoles have been found to be required in various extensions of the standard model of particle physics. Dudley and Finley [8], carried out the separation of variables for all real, massless, single spin field equations with $s=0, \frac{1}{2}, 1,2$ on the seven-parameter class of Petrov type-D solutions of Einstein-Maxwell equations found by Plebanski and Demianski [7]. The seven parameters include $M, a, Q_{e}$ and $Q_{m}$ (The coordinates are not of Boyer-Lindquist type). Since the fields are real, these results apply only to neutral particles. In this paper, we use the tetrad formalism to show that the Dirac equation for massive, charged fermions remains separable when magnetic charge is added to the black hole, i.e. in the dyonic Kerr-Newman case; and we present the separated radial and angular equations. We anticipate using this separability in a thought experiment to test the cosmic censorship conjecture by considering a massive, charged Dirac field on a dyonic black hole, i.e. the Dirac-field analogue of work done in [9]. 


\section{The Tetrad Formalism}

The equivalence principle tells us that the laws of Physics, as written in Minkowski space, are also valid in a freely falling frame on a curved space, i.e. a local Lorentz coordinate system (LLCS). This principle can be utilized to construct the tetrad formalism \&, a prescription for writing the laws of Physics on curved spaces. We erect at each point in spacetime, four vectors $V_{a}{ }^{\mu}$, the "basis vectors of a LLCS". Here, the latin indices from the early part of the alphabet label the coordinates in the LLCS, and the greek indices, the spacetime coordinates. The set of four $V_{a}$ 's, the tetrad or vierbein, is usually chosen to be orthonormal:

$$
g_{\mu \nu} V_{a}^{\mu} V_{b}^{\nu}=\eta_{a b}
$$

The LLCS indices are raised and lowered with the Minkowski tensor $\eta_{a b}$ (we use $\eta_{a b}=\operatorname{diag}(-1,1,1,1)$ ) and spacetime indices with the metric $g_{\mu \nu}$; the tetrad satisfies

$$
\eta_{a b} V_{\mu}^{a} V_{\nu}^{b}=g_{\mu \nu}
$$

We can write down components of physical fields in the LLCS:

$$
A_{c d \cdots}^{a b \cdots}=V_{\mu}^{a} V_{\nu}^{b} V_{c}^{\sigma} V_{d}^{\eta} \cdots A_{\sigma \eta \cdots}^{\mu \nu \cdots}
$$

These objects $A_{c d \cdots}^{a b \cdots}$ are scalars with respect to spacetime operations, and tensors in the LLCS, the number of the covariant and contravariant indices being the same as for the original object $A_{\sigma \eta \cdots}^{\mu \nu \cdots}$

In terms of these components, we want to write down laws of Physics in the LLCS, in their Minkowskian form. We also want the laws to be invariant under local Lorentz transformations, i.e. different tetrad choices. This leads us to define the coordinate-scalar, Lorentz-vector 'derivative'

$$
\mathcal{D}_{a}=V_{a}^{\mu} \partial_{\mu}+\frac{1}{2} V_{a}^{\mu} V_{b}^{\nu} V_{c \nu ; \mu} \Sigma^{b c}
$$

where $\Sigma^{\alpha \beta}$ are generators of the Lorentz group in the representation associated with the field $\mathcal{D}_{a}$ is acting on; and the semicolon denotes the (metric-)covariant derivative ${ }^{3}$.

Now the prescription is: We take the Minkowskian form of the relevant equation, replace each term by its LLCS equivalent via eqn. (3) and each derivative by its LLCS equivalent

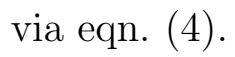

\footnotetext{
${ }^{2}$ See, e.g. Weinberg [10], Section 12.5, Birrell \& Davies [11], Section 3.8, Chandrasekhar [12, $\S 7$.

${ }^{3}$ Weinberg [10] and Birell \& Davies [11] give -incorrectly- an ordinary derivative here.
} 


\section{The Dirac Equation on the dyonic black hole metric}

The Dirac Equation in flat space with a background electromagnetic field is

$$
i \gamma^{a}\left(\partial_{a}+i e A_{a}\right) \Psi=\mu \Psi
$$

where $\Psi$ is a four-component spinor, $\mu$ the mass and $e$ the charge of the field quantum, $A$ the vector-potential of the electromagnetic field and the constant $\gamma$-matrices satisfy

$$
\left\{\gamma^{a}, \gamma^{b}\right\}=-2 \eta^{a b}
$$

We will be using $\gamma$-matrices of the form

$$
\gamma^{0}=\left(\begin{array}{cc}
0 & \sigma^{0} \\
\sigma^{0} & 0
\end{array}\right), \quad \gamma^{i}=\left(\begin{array}{cc}
0 & -\sigma^{i} \\
\sigma^{i} & 0
\end{array}\right)
$$

where the $\sigma$-matrices are the identity matrix and the Pauli matrice s:

$$
\sigma^{0}=\left(\begin{array}{ll}
1 & 0 \\
0 & 1
\end{array}\right), \quad \sigma^{1}=\left(\begin{array}{ll}
0 & 1 \\
1 & 0
\end{array}\right), \quad \sigma^{2}=\left(\begin{array}{rr}
0 & -i \\
i & 0
\end{array}\right), \quad \sigma^{3}=\left(\begin{array}{rr}
1 & 0 \\
0 & -1
\end{array}\right)
$$

For a spin- $\frac{1}{2}$ field, the $\Sigma$-matrices are given by

$$
\Sigma^{b c}=-\frac{1}{4}\left[\gamma^{b}, \gamma^{c}\right] .
$$

To get the Dirac Equation in curved space, we follow the prescription and make the replacements (3, , $^{2}$ :

$$
\left.i\left[\gamma^{a}\right]_{k}^{j} V_{a}^{\mu} \partial_{\mu} \Psi_{j}+\frac{1}{2} i\left[\gamma^{a}\right]_{k}^{l} V_{a}^{\mu} V_{b}{ }^{\nu} V_{c \nu ; \mu}\left[\Sigma^{b c}\right]_{l}^{j} \Psi_{j}-e\left[\gamma^{a}\right]_{k}^{j} V_{a}^{\mu} A_{\mu}\right) \Psi_{j}=\mu \Psi_{k}
$$

where we have written out the spinor indices $j, k, l$ explicitly.

The metric of the dyonic black hole is

$$
\begin{aligned}
d s^{2}=g_{\mu \nu} d x^{\mu} d x^{\nu} & =\frac{a^{2} \sin ^{2} \theta-\Delta}{\rho^{2}} d t^{2}+\frac{\left(r^{2}+a^{2}\right)^{2}-\Delta a^{2} \sin ^{2} \theta}{\rho^{2}} \sin ^{2} \theta d \phi^{2} \\
& +2 \frac{\Delta-\left(r^{2}+a^{2}\right)}{\rho^{2}} a \sin ^{2} \theta d t d \phi+\frac{\rho^{2}}{\Delta} d r^{2}+\rho^{2} d \theta^{2}
\end{aligned}
$$

where

$$
\begin{aligned}
& \rho^{2}=r^{2}+a^{2} \cos ^{2} \theta \\
& \Delta=r^{2}-2 M r+a^{2}+Q_{e}{ }^{2}+Q_{m}{ }^{2}
\end{aligned}
$$

\footnotetext{
${ }^{4}$ For derivations in Boyer-Lindquist coordinates, see [9] and [13].
} 
with the vector potential

$$
\begin{aligned}
A_{t} & =-Q_{e} \frac{r}{\rho^{2}}+Q_{m} \frac{a \cos \theta}{\rho^{2}} \\
A_{r} & =A_{\theta}=0 \\
A_{\phi} & =Q_{e} \frac{\operatorname{ar} \sin ^{2} \theta}{\rho^{2}}+Q_{m}\left[ \pm 1-\cos \theta \frac{r^{2}+a^{2}}{\rho^{2}}\right] .
\end{aligned}
$$

and we are using Boyer-Lindquist coordinates.

Of course, the vector potential is unique only up to a gauge transformation, and the magnetic part of $A_{\phi}$ contains a string singularity. The two signs in that term correspond to the two gauges we will be using. The upper-sign term puts the string along the negative $z$-axis $(\theta=\pi)$ and will be used when $0 \leq \theta \leq \pi / 2$, the lower-sign term puts it along the positive $z$-axis $(\theta=0)$ and will be used when $\pi / 2<\theta \leq \pi$. Therefore, the wavefunction is also gauge transformed across the equator, and picks up a factor of $e^{2 i e Q_{m} \phi}$ passing from north to south. This matching of boundary conditions ensures that the problem can be expressed meaningfully without strings of diverging vector potential. Such a wavefunction is called a section [14].

We will derive separated equations first using a simple tetrad that we call 'canonical'. For purposes of comparison at the appropriate limit with Chandrasekhar [4], we will repeat the calculation using a more complicated tetrad, calling it 'Kinnersley-type'. The labels $(C)$ and $(K)$ will be used for variables whenever it is not obvious which case they belong to or for emphasis.

\subsection{The Canonical Tetrad}

We use the generalization of the simple 'canonical' tetrad emphasized by Carter, relabeling the vectors to be able to compare with Carter \& McLenaghan [19] in the limit $Q_{m}=0$ :

$$
\begin{aligned}
V^{(C) 0} & =\frac{\delta}{\rho}\left(d t-a \sin ^{2} \theta d \phi\right) \\
V^{(C) 1} & \left.=\frac{\sin \theta}{\rho}\left[-a d t+\left(r^{2}+a^{2}\right) d \phi\right)\right] \\
V^{(C) 2} & =\rho d \theta \\
V^{(C) 3} & =\frac{\rho}{\delta} d r
\end{aligned}
$$

where $\delta=\sqrt{\Delta}$ and $\rho$ was defined above via its square.

Making all the necessary substitutions into eq. (10), we get the four coupled components 
of the Dirac equation f́ on the dyon black hole metric:

$$
\begin{aligned}
& {\left[\frac{\left(r^{2}+a^{2}\right)}{\rho \delta} \partial_{t}-\frac{\delta}{\rho} \partial_{r}+\frac{a}{\rho \delta} \partial_{\phi}-\frac{\delta}{2 \rho \bar{\rho}^{*}}-\frac{(r-M)}{2 \rho \delta}+i e \frac{-Q_{e} r \pm a Q_{m}}{\rho \delta}\right] \Psi_{2}^{(C)}} \\
& +\left[-\frac{a \sin \theta}{\rho} \partial_{t}+\frac{i}{\rho} \partial_{\theta}-\frac{1}{\rho \sin \theta} \partial_{\phi}-\frac{a \sin \theta}{2 \rho \bar{\rho}^{*}}+\frac{i}{2 \rho} \cot \theta-i e Q_{m} \frac{ \pm 1-\cos \theta}{\rho \sin \theta}\right] \Psi_{3}^{(C)}=-i \mu \Psi_{0}^{(C)} \\
& {\left[-\frac{a \sin \theta}{\rho} \partial_{t}-\frac{i}{\rho} \partial_{\theta}-\frac{1}{\rho \sin \theta} \partial_{\phi}+\frac{a \sin \theta}{2 \rho \bar{\rho}^{*}}-\frac{i}{2 \rho} \cot \theta-i e Q_{m} \frac{ \pm 1-\cos \theta}{\rho \sin \theta}\right] \Psi_{2}^{(C)}} \\
& +\left[\frac{\left(r^{2}+a^{2}\right)}{\rho \delta} \partial_{t}+\frac{\delta}{\rho} \partial r+\frac{a}{\rho \delta} \partial \phi+\frac{\delta}{2 \rho \bar{\rho}^{*}}+\frac{(r-M)}{2 \rho \delta}+i e \frac{-Q_{e} r \pm a Q_{m}}{\rho \delta}\right] \Psi_{3}^{(C)}=-i \mu \Psi_{1}^{(C)} \\
& {\left[\frac{\left(r^{2}+a^{2}\right)}{\rho \delta} \partial_{t}+\frac{\delta}{\rho} \partial r+\frac{a}{\rho \delta} \partial \phi+\frac{\delta}{2 \rho \bar{\rho}}+\frac{(r-M)}{2 \rho \delta}+i e \frac{-Q_{e} r \pm a Q_{m}}{\rho \delta}\right] \Psi_{0}^{(C)}} \\
& +\left[\frac{a \sin \theta}{\rho} \partial_{t}-\frac{i}{\rho} \partial_{\theta}+\frac{1}{\rho \sin \theta} \partial_{\phi}-\frac{a \sin \theta}{2 \rho \bar{\rho}}-\frac{i}{2 \rho} \cot \theta+i e Q_{m} \frac{ \pm 1-\cos \theta}{\rho \sin \theta}\right] \Psi_{1}^{(C)}=-i \mu \Psi_{2}^{(C)} \\
& {\left[\frac{a \sin \theta}{\rho} \partial_{t}+\frac{i}{\rho} \partial_{\theta}+\frac{1}{\rho \sin \theta} \partial_{\phi}+\frac{a \sin \theta}{2 \rho \bar{\rho}}+\frac{i}{2 \rho} \cot \theta+i e Q_{m} \frac{ \pm 1-\cos \theta}{\rho \sin \theta}\right] \Psi_{0}^{(C)}} \\
& +\left[\frac{\left(r^{2}+a^{2}\right)}{\rho \delta} \partial_{t}-\frac{\delta}{\rho} \partial r+\frac{a}{\rho \delta} \partial \phi-\frac{\delta}{2 \rho \bar{\rho}}-\frac{(r-M)}{2 \rho \delta}+i e \frac{-Q_{e} r \pm a Q_{m}}{\rho \delta}\right] \Psi_{1}^{(C)}=-i \mu \Psi_{3}^{(C)}
\end{aligned}
$$

where

$$
\bar{\rho}=r+i a \cos \theta, \quad \bar{\rho}^{*}=r-i a \cos \theta, \quad \bar{\rho} \bar{\rho}^{*}=\rho^{2}
$$

\subsection{The 'Kinnersley-type' Tetrad}

For sake of comparison with Chandrasekhar $\llbracket$, in the limit $Q_{e}=0, Q_{m}=0$, we write down the Dirac Equation using the generalization of the Kinnersley-type tetrad corresponding to the null tetrad used by him:

$$
\begin{aligned}
V^{(K) 0} & =\frac{1}{\sqrt{2}}\left[\left(1+\frac{\Delta}{2 \rho^{2}}\right) d t+\left(\frac{1}{2}-\frac{\rho^{2}}{\Delta}\right) d r-\left(1+\frac{\Delta}{2 \rho^{2}}\right) a \sin ^{2} \theta d \phi\right] \\
V^{(K) 1} & =-\frac{a^{2} \cos \theta \sin \theta}{\rho^{2}} d t+r d r+\frac{a\left(a^{2}+r^{2}\right) \cos \theta \sin \theta}{\rho^{2}} d \phi \\
V^{(K) 2} & =\frac{a r \sin \theta}{\rho^{2}} d t+a \cos \theta d \theta-\frac{r\left(a^{2}+r^{2}\right) \sin \theta}{\rho^{2}} d \phi \\
V^{(K) 3} & =\frac{1}{\sqrt{2}}\left[\left(-1+\frac{\Delta}{2 \rho^{2}}\right) d t+\left(\frac{1}{2}+\frac{\rho^{2}}{\Delta}\right) d r+\left(1-\frac{\Delta}{2 \rho^{2}}\right) a \sin ^{2} \theta d \phi\right]
\end{aligned}
$$

${ }^{5}$ The calculations for going from eqn. (10) to eqns. (14a $14 \mathrm{~d}$ ) or eqns. (16a $16 \mathrm{~d}$ ) have been performed using the Mathematica symbolic mathematics software, and the 'tetrad' package [15]. 
Once again, making necessary get four coupled components of the Dirac equation on the dyon black hole metric; for the Kinnersley-type tetrad:

$$
\begin{aligned}
& {\left[\frac{\left(r^{2}+a^{2}\right)}{\sqrt{2} \rho^{2}} \partial_{t}-\frac{\Delta}{\sqrt{2} \rho^{2}} \partial_{r}+\frac{a}{\sqrt{2} \rho^{2}} \partial_{\phi}-\frac{(r-M)}{\sqrt{2} \rho^{2}}+\frac{i e}{\sqrt{2}}\left(\frac{ \pm a Q_{m}-Q_{e} r}{\rho^{2}}\right)\right] \Psi_{2}^{(K)}} \\
& +\left[-\frac{i a \sin \theta}{\bar{\rho}} \partial_{t}-\frac{1}{\bar{\rho}} \partial_{\theta}-\frac{i}{\bar{\rho} \sin \theta} \partial_{\phi}-\left(\frac{i a \sin \theta}{\rho^{2}}+\frac{1}{2 \bar{\rho}} \cot \theta\right)+e Q_{m}\left(\frac{ \pm 1-\cos \theta}{\bar{\rho} \sin \theta}\right)\right] \Psi_{3}^{(K)}=-i \mu \Psi_{0}^{(K)} \\
& {\left[\frac{i a \sin \theta}{\bar{\rho}^{*}} \partial_{t}-\frac{1}{\bar{\rho}^{*}} \partial_{\theta}+\frac{i}{\bar{\rho}^{*} \sin \theta} \partial_{\phi}-\frac{1}{2 \bar{\rho}^{*}} \cot \theta-e Q_{m}\left(\frac{ \pm 1-\cos \theta}{\bar{\rho}^{*} \sin \theta}\right)\right] \Psi_{2}^{(K)}} \\
& +\left[\sqrt{2} \frac{\left(r^{2}+a^{2}\right)}{\Delta} \partial_{t}+\sqrt{2} \partial_{r}+\sqrt{2} \frac{a}{\Delta} \partial_{\phi}+\sqrt{2} \frac{1}{\bar{\rho}^{*}}+i e \sqrt{2}\left(\frac{ \pm a Q_{m}-Q_{e} r}{\Delta}\right)\right] \Psi_{3}^{(K)}=-i \mu \Psi_{1}^{(K)} \\
& {\left[\sqrt{2} \frac{\left(r^{2}+a^{2}\right)}{\Delta} \partial_{t}+\sqrt{2} \partial_{r}+\sqrt{2} \frac{a}{\Delta} \partial_{\phi}+\sqrt{2} \frac{1}{\bar{\rho}}+i e \sqrt{2}\left(\frac{ \pm a Q_{m}-Q_{e} r}{\Delta}\right)\right] \Psi_{0}^{(K)}} \\
& +\left[\frac{i a \sin \theta}{\bar{\rho}} \partial_{t}+\frac{1}{\bar{\rho}} \partial_{\theta}+\frac{i}{\bar{\rho} \sin \theta} \partial_{\phi}+\frac{1}{2 \bar{\rho}} \cot \theta-e Q_{m}\left(\frac{ \pm 1-\cos \theta}{\bar{\rho} \sin \theta}\right)\right] \Psi_{1}^{(K)}=-i \mu \Psi_{2}^{(K)} \\
& {\left[-\frac{i a \sin \theta}{\bar{\rho}^{*}} \partial_{t}+\frac{1}{\bar{\rho}^{*}} \partial_{\theta}-\frac{i}{\bar{\rho}^{*} \sin \theta} \partial_{\phi}+\left(-\frac{i a \sin \theta}{\rho^{2}}+\frac{1}{2 \bar{\rho}^{*}} \cot \theta\right)+e Q_{m}\left(\frac{ \pm 1-\cos \theta}{\bar{\rho}^{*} \sin \theta}\right)\right] \Psi_{0}^{(K)}} \\
& +\left[\frac{\left(r^{2}+a^{2}\right)}{\sqrt{2} \rho^{2}} \partial_{t}-\frac{\Delta}{\sqrt{2} \rho^{2}} \partial_{r}+\frac{a}{\sqrt{2} \rho^{2}} \partial_{\phi}-\frac{r-M}{\sqrt{2} \rho^{2}}+\frac{i e}{\sqrt{2}}\left(\frac{ \pm a Q_{m}-Q_{e} r}{\rho^{2}}\right)\right] \Psi_{1}^{(K)}=-i \mu \Psi_{3}^{(K)}
\end{aligned}
$$

\section{The Separation}

To separate eqns. (14a-14d ) or (16a,16d), we assign the standard time- and azimuthal dependence to $\Psi$, with the above-mentioned gauge transformation across the equiatorial plane:

$$
\Psi=e^{-i \omega t} e^{i\left(m \mp e Q_{m}\right) \phi} \psi
$$

\subsection{The Canonical Tetrad}

Substituting eqn. (17) into (14a-14d) gives, after some algebra,

$$
\begin{aligned}
-\frac{\delta}{\rho}\left(\mathcal{D}_{+}+\frac{1}{2 \bar{\rho}^{*}}\right) \psi_{2}+\frac{i}{\rho}\left(\mathcal{L}_{-}+\frac{i a \sin \theta}{2 \bar{\rho}^{*}}\right) \psi_{3} & =-i \mu \psi_{0} \\
-\frac{i}{\rho}\left(\mathcal{L}_{+}+\frac{i a \sin \theta}{2 \bar{\rho}^{*}}\right) \psi_{2}+\frac{\delta}{\rho}\left(\mathcal{D}_{-}+\frac{1}{2 \bar{\rho}^{*}}\right) \psi_{3} & =-i \mu \psi_{1} \\
\frac{\delta}{\rho}\left(\mathcal{D}_{-}+\frac{1}{2 \bar{\rho}}\right) \psi_{0}-\frac{i}{\rho}\left(\mathcal{L}_{-}-\frac{i a \sin \theta}{2 \bar{\rho}}\right) \psi_{1} & =-i \mu \psi_{2} \\
\frac{i}{\rho}\left(\mathcal{L}_{+}-\frac{i a \sin \theta}{2 \bar{\rho}}\right) \psi_{0}-\frac{\delta}{\rho}\left(\mathcal{D}_{+}+\frac{1}{2 \bar{\rho}}\right) \psi_{1} & =-i \mu \psi_{3}
\end{aligned}
$$


where $\mathcal{D}_{ \pm}^{(C)}$ and $\mathcal{L}_{ \pm}^{(C)}$ are purely radial and purely angular, respectively:

$$
\begin{gathered}
\mathcal{D}_{+}^{(C)}=\partial_{r}+i \frac{\left(r^{2}+a^{2}\right) \omega+e Q_{e} r-m a}{\Delta}+\frac{(r-M)}{2 \Delta} \\
\mathcal{D}_{-}^{(C)}=\partial_{r}-i \frac{\left(r^{2}+a^{2}\right) \omega+e Q_{e} r-m a}{\Delta}+\frac{(r-M)}{2 \Delta} \\
\mathcal{L}_{+}^{(C)}=\partial_{\theta}-a \omega \sin \theta+\frac{m}{\sin \theta}+\left(\frac{1}{2}+e Q_{m}\right) \cot \theta \\
\mathcal{L}_{-}^{(C)}=\partial_{\theta}+a \omega \sin \theta-\frac{m}{\sin \theta}+\left(\frac{1}{2}-e Q_{m}\right) \cot \theta
\end{gathered}
$$

Defining $\psi_{0}=f_{0} / \sqrt{\bar{\rho}}, \psi_{1}=f_{1} / \sqrt{\bar{\rho}}, \psi_{2}=f_{2} / \sqrt{\bar{\rho}^{*}}$, and $\psi_{3}=f_{3} / \sqrt{\bar{\rho}^{*}}$; multiplying eqns. (18a) and (18b) by $\rho \sqrt{\bar{\rho}^{*}}$, eqns. (18d) and (18d) by $\rho \sqrt{\bar{\rho}}$, we get

$$
\begin{aligned}
-\delta \mathcal{D}_{+} f_{2}+i \mathcal{L}_{-} f_{3} & =-i \mu(r-i a \cos \theta) f_{0} \\
\delta \mathcal{D}_{-} f_{3}-i \mathcal{L}_{+} f_{2} & =-i \mu(r-i a \cos \theta) f_{1} \\
\delta \mathcal{D}_{-} f_{0}-i \mathcal{L}_{-} f_{1} & =-i \mu(r+i a \cos \theta) f_{2} \\
-\delta \mathcal{D}_{+} f_{1}+i \mathcal{L}_{+} f_{0} & =-i \mu(r+i a \cos \theta) f_{3}
\end{aligned}
$$

The structure of eqns. (21a 21d) suggests that they can be separated by the substitutions

$$
\begin{aligned}
& f_{0}=R_{-} S_{+} \quad ; \quad f_{1}=R_{+} S_{-} \\
& f_{2}=R_{+} S_{+} \quad ; \quad f_{3}=R_{-} S_{-}
\end{aligned}
$$

which gives

$$
\begin{aligned}
& \delta \mathcal{D}_{+} R_{+}-i \mu r R_{-}=\lambda_{0} R_{-} \quad ; \quad i \mathcal{L}_{-} S_{-}+\mu a \cos \theta S_{+}=\lambda_{0} S_{+} \\
& \delta \mathcal{D}_{-} R_{-}+i \mu r R_{+}=\lambda_{1} R_{+} \quad ; \quad i \mathcal{L}_{+} S_{+}-\mu a \cos \theta S_{-}=\lambda_{1} S_{-} \\
& \delta \mathcal{D}_{-} R_{-}+i \mu r R_{+}=\lambda_{2} R_{+} \quad ; \quad i \mathcal{L}_{-} S_{-}+\mu a \cos \theta S_{+}=\lambda_{2} S_{+} \\
& \delta \mathcal{D}_{+} R_{+}-i \mu r R_{-}=\lambda_{3} R_{-} \quad ; \quad i \mathcal{L}_{+} S_{+}-\mu a \cos \theta S_{-}=\lambda_{3} S_{-}
\end{aligned}
$$

The consistency of these equations requires

$$
\lambda_{0}=\lambda_{3}=\lambda_{1}=\lambda_{2}=\lambda
$$

and we are left with

$$
\begin{aligned}
& \delta \mathcal{D}_{+}^{(C)} R_{+}^{(C)}-i \mu r R_{-}^{(C)}=\lambda R_{-}^{(C)} \\
& \delta \mathcal{D}_{-}^{(C)} R_{-}^{(C)}+i \mu r R_{+}^{(C)}=\lambda R_{+}^{(C)}
\end{aligned}
$$


and

$$
\begin{aligned}
& \mathcal{L}_{+}^{(C)} S_{+}^{(C)}+i \mu a \cos \theta S_{-}^{(C)}=-i \lambda S_{-}^{(C)} \\
& \mathcal{L}_{-}^{(C)} S_{-}^{(C)}-i \mu a \cos \theta S_{+}^{(C)}=-i \lambda S_{+}^{(C)}
\end{aligned}
$$

Or, by combining pairs, we can get decoupled $2^{\text {nd }}$ order equations

$$
\begin{gathered}
{\left[\delta \mathcal{D}_{-}^{(C)} \delta \mathcal{D}_{+}^{(C)}-\frac{i \mu \Delta}{(\lambda+i \mu r)} \mathcal{D}_{+}^{(C)}-\left(\lambda^{2}+\mu^{2} r^{2}\right)\right] R_{+}^{(C)}=0} \\
{\left[\delta \mathcal{D}_{+}^{(C)} \delta \mathcal{D}_{-}^{(C)}+\frac{i \mu \Delta}{(\lambda-i \mu r)} \mathcal{D}_{-}^{(C)}-\left(\lambda^{2}+\mu^{2} r^{2}\right)\right] R_{-}^{(C)}=0} \\
{\left[\mathcal{L}_{-}^{(C)} \mathcal{L}_{+}^{(C)}+\frac{\mu a \sin \theta}{\lambda+\mu a \sin \theta} \mathcal{L}_{+}^{(C)}+\left(\lambda^{2}-\mu^{2} a^{2} \cos ^{2} \theta\right)\right] S_{+}^{(C)}=0} \\
{\left[\mathcal{L}_{+}^{(C)} \mathcal{L}_{-}^{(C)}-\frac{\mu a \sin \theta}{\lambda-\mu a \cos \theta} \mathcal{L}_{-}^{(C)}+\left(\lambda^{2}-\mu^{2} a^{2} \cos ^{2} \theta\right)\right] S_{-}^{(C)}=0}
\end{gathered}
$$

\subsection{The Kinnersley-type Tetrad}

The same time- and azimuthal dependence (17), when substituted into eqns. (16a-16d) gives

$$
\begin{aligned}
\frac{\Delta}{\sqrt{2} \rho^{2}} \mathcal{D}_{+} \psi_{2}+\frac{1}{\bar{\rho}}\left(\mathcal{L}_{+}+\frac{i a \sin \theta}{\bar{\rho}^{*}}\right) \psi_{3} & =i \mu \psi_{0} \\
\sqrt{2}\left(\mathcal{D}_{-}+\frac{1}{\bar{\rho}^{*}}\right) \psi_{3}-\frac{1}{\bar{\rho}^{*}} \mathcal{L}_{-} \psi_{2} & =-i \mu \psi_{1} \\
\sqrt{2}\left(\mathcal{D}_{-}+\frac{1}{\bar{\rho}}\right) \psi_{0}+\frac{1}{\bar{\rho}} \mathcal{L}_{+} \psi_{1} & =-i \mu \psi_{2} \\
\frac{\Delta}{\sqrt{2} \rho^{2}} \mathcal{D}_{+} \psi_{1}-\frac{1}{\bar{\rho}^{*}}\left(\mathcal{L}_{-}-\frac{i a \sin \theta}{\bar{\rho}}\right) \psi_{0} & =i \mu \psi_{3}
\end{aligned}
$$

the radial and angular operators $\mathcal{D}_{ \pm}^{(K)}$ and $\mathcal{L}_{ \pm}^{(K)}$ being

$$
\begin{aligned}
\mathcal{D}_{+}^{(K)} & =\partial_{r}+\frac{i}{\Delta}\left[\left(r^{2}+a^{2}\right) \omega-m a+e Q_{e} r\right]+\frac{(r-M)}{\Delta} \\
\mathcal{D}_{-}^{(K)}= & \partial_{r}-\frac{i}{\Delta}\left[\left(r^{2}+a^{2}\right) \omega-m a+e Q_{e} r\right] \\
\mathcal{L}_{+}^{(K)} & =\partial_{\theta}+a \omega \sin \theta-\frac{m}{\sin \theta}+\left(\frac{1}{2}+e Q_{m}\right) \cot \theta \\
\mathcal{L}_{-}^{(K)} & =\partial_{\theta}-a \omega \sin \theta+\frac{m}{\sin \theta}+\left(\frac{1}{2}-e Q_{m}\right) \cot \theta
\end{aligned}
$$


Multiplying eqns. (29a and (29d) by $\rho^{2}=\bar{\rho} \bar{\rho}^{*}$, eq. (29b) by $\bar{\rho}^{*}$, eq. (29c) by $\bar{\rho}$; defining $F_{0}=\bar{\rho} \psi_{0}, F_{1}=\psi_{1}, F_{2}=\psi_{2}$, and $F_{3}=\bar{\rho}^{*} \psi_{3}$, we get

$$
\begin{aligned}
& \frac{\Delta}{\sqrt{2}} \mathcal{D}_{+} F_{2}+\mathcal{L}_{+} F_{3}=i \mu(r-i a \cos \theta) F_{0} \\
& \sqrt{2} \mathcal{D}_{-} F_{3}-\mathcal{L}_{-} F_{2}=-i \mu(r-i a \cos \theta) F_{1} \\
& \sqrt{2} \mathcal{D}_{-} F_{0}+\mathcal{L}_{+} F_{1}=-i \mu(r+i a \cos \theta) F_{2} \\
& \frac{\Delta}{\sqrt{2}} \mathcal{D}_{+} F_{1}-\mathcal{L}_{-} F_{0}=i \mu(r+i a \cos \theta) F_{3}
\end{aligned}
$$

Again, the eqns. $32 \mathrm{a} 32 \mathrm{~d}$ can be separated by

$$
\begin{aligned}
& F_{0}=R_{-} S_{+} \quad ; \quad F_{1}=R_{+} S_{-} \\
& F_{2}=R_{+} S_{+} \quad ; \quad F_{3}=R_{-} S_{-}
\end{aligned}
$$

which gives

$$
\begin{array}{ll}
\frac{\Delta}{\sqrt{2}} \mathcal{D}_{+} R_{+}-i \mu r R_{-}=\lambda_{0} R_{-} \quad ; \quad \mathcal{L}_{+} S_{-}-\mu a \cos \theta S_{+}=-\lambda_{0} S_{+} \\
\sqrt{2} \mathcal{D}_{-} R_{-}+i \mu r R_{+}=\lambda_{1} R_{+} \quad ; \quad \mathcal{L}_{-} S_{+}-\mu a \cos \theta S_{-}=\lambda_{1} S_{-} \\
\sqrt{2} \mathcal{D}_{-} R_{-}+i \mu r R_{+}=\lambda_{2} R_{+} \quad ; \quad \mathcal{L}_{+} S_{-}-\mu a \cos \theta S_{+}=-\lambda_{2} S_{+} \\
\frac{\Delta}{\sqrt{2}} \mathcal{D}_{+} R_{+}-i \mu r R_{-}=\lambda_{3} R_{-} \quad ; \quad \mathcal{L}_{-} S_{+}-\mu a \cos \theta S_{-}=\lambda_{3} S_{-}
\end{array}
$$

as before,

$$
\lambda_{0}=\lambda_{3}=\lambda_{1}=\lambda_{2}=\lambda^{\prime}
$$

therefore

$$
\begin{gathered}
\frac{\Delta}{\sqrt{2}} \mathcal{D}_{+}^{(K)} R_{+}^{(K)}-i \mu r R_{-}^{(K)}=\lambda^{\prime} R_{-}^{(K)} \\
\sqrt{2} \mathcal{D}_{-}^{(K)} R_{-}^{(K)}+i \mu r R_{+}^{(K)}=\lambda^{\prime} R_{+}^{(K)} \\
\mathcal{L}_{+}^{(K)} S_{-}^{(K)}-\mu a \cos \theta S_{+}^{(K)}=-\lambda^{\prime} S_{+}^{(K)} \\
\mathcal{L}_{-}^{(K)} S_{+}^{(K)}-\mu a \cos \theta S_{-}^{(K)}=\lambda^{\prime} S_{-}^{(K)}
\end{gathered}
$$

The corresponding decoupled $2^{\text {nd }}$ order equations are

$$
\begin{aligned}
{\left[\Delta \mathcal{D}_{+}^{(K)} \mathcal{D}_{-}^{(K)}+\frac{i \mu \Delta}{\left(\lambda^{\prime}-i \mu r\right)} \mathcal{D}_{-}^{(K)}-\left(\lambda^{\prime 2}+\mu^{2} r^{2}\right)\right] R_{-}^{(K)} } & =0 \\
{\left[\mathcal{D}_{-}^{(K)} \Delta \mathcal{D}_{+}^{(K)}-\frac{i \mu}{\left(\lambda^{\prime}+i \mu r\right)} \Delta \mathcal{D}_{+}^{(K)}-\left(\lambda^{\prime 2}+\mu^{2} r^{2}\right)\right] R_{+}^{(K)} } & =0
\end{aligned}
$$




$$
\begin{aligned}
& {\left[\mathcal{L}_{+}^{(K)} \mathcal{L}_{-}^{(K)}+\frac{\mu a \sin \theta}{\lambda^{\prime}+\mu a \cos \theta} \mathcal{L}_{-}^{(K)}+\left(\lambda^{\prime 2}-\mu^{2} a^{2} \cos ^{2} \theta\right)\right] S_{+}^{(K)}=0} \\
& {\left[\mathcal{L}_{-}^{(K)} \mathcal{L}_{+}^{(K)}-\frac{\mu a \sin \theta}{\lambda^{\prime}-\mu a \cos \theta} \mathcal{L}_{+}^{(K)}+\left(\lambda^{\prime 2}-\mu^{2} a^{2} \cos ^{2} \theta\right)\right] S_{-}^{(K)}=0}
\end{aligned}
$$

\section{Comparison with previous results}

Both papers that we compare our results against use the Newman-Penrose formalism. For the simpler 'canonical tetrad', our results (eqns.25a-28b) agree with Carter \& McLenaghan [19] in the limit $Q_{m}=0$, with $\mathcal{D}_{ \pm}^{(C)} \rightarrow \mathcal{D}_{ \pm \frac{1}{2}}, \mathcal{L}_{ \pm}^{(C)} \rightarrow \mathcal{L}_{ \pm \frac{1}{2}}, R_{ \pm}^{(C)} \rightarrow X_{ \pm \frac{1}{2}}, S_{+}^{(C)} \rightarrow Y_{\frac{1}{2}}$, $S_{-}^{(C)} \rightarrow-Y_{-\frac{1}{2}}, \lambda \rightarrow \sqrt{2} \lambda, \mu \rightarrow \sqrt{2} \mu$. For the Kinnersley-type tetrad, the equations (36a39b) agree with Chandrasekhar [12] in the limit $e=Q_{e}=Q_{m}=0$, with $\mathcal{D}_{+}^{(K)} \rightarrow \mathcal{D}_{\frac{1}{2}}^{\dagger}$, $\mathcal{D}_{-}^{(K)} \rightarrow \mathcal{D}_{0}, \mathcal{L}_{+}^{(K)} \rightarrow \mathcal{L}_{\frac{1}{2}}^{\dagger}, \mathcal{L}_{-}^{(K)} \rightarrow \mathcal{L}_{\frac{1}{2}}, S_{ \pm}^{(K)} \rightarrow S_{ \pm \frac{1}{2}}, R_{+}^{(K)} \rightarrow R_{+\frac{1}{2}}, R_{-}^{(K)} \rightarrow-R_{-\frac{1}{2}}, \lambda^{\prime} \rightarrow-\lambda$. A difference with the $\hat{Q}_{m}=0$ case is that $m$ can now also take on half-integer values: To make the wavefunctions (17) single-valued, both $m+e Q_{m}$ and $m-e Q_{m}$ have to be integers, which makes $m$ and $e Q_{m}$ integers or half-integers, the latter requirement being, of course, the well-known Dirac quantization condition. Since the explicit forms of equations (27a 28b) and (38a $39 \mathrm{~b})$ are not very illuminating, we leave them in their present form. Unfortunately, the angular equations (28a, 280, 39a. 39b), unlike in the simpler scalar case [16], are not of the Sturm-Liouville form, therefore we cannot make a statement about the completeness of the solutions.

The Chandrasekhar result has been also generalized to other cases [17], [22], [23], [29], the mathematical structure of the problem has been investigated [18], [19], [22], [21], [22], [22], [24], [25] and the solutions studied [26], [27], [28]. Other approaches to the problem of separation of variables in the Dirac Equation on curved spaces include the Stäckel Space method [30] and the "algebraic" method [31].

\section{Acknowledgements :}

I am grateful to V. Moncrief for valuable discussions and helpful suggestions. This research was partially supported by NSF grant PHY-8903939 to Yale University.

\section{References}

[1] D. Brill and J. A. Wheeler, Rev. Mod. Phys. 29645 (1957).

[2] S. A. Teukolsky, Astrophysical J. 185, 635 (1973). 
[3] W. Unruh, Phys. Rev. Lett. 31, 1265 (1973)

[4] S. Chandrasekhar, Proc. R. Soc. A 349, 571 (1976); see S. Chandrasekhar, Proc. R. Soc. A 350, 564 (1976) for errata; also see [12], $§ 104$.

[5] D. N. Page, Phys. Rev. D 14, 1509, (1976).

[6] N. Toop, preprint, D.A.M.T.P., Cambridge (1976).

[7] J. F. Plebanski and M. Demianski, Ann. Phys. 98, 98 (1976).

[8] A. L. Dudley and J. D. Finley, J.Math. Phys. 20, 311 (1979).

[9] İ. Semiz, Class. Quant. Grav. 7, 353 (1990).

[10] S. Weinberg, Gravitation and Cosmology, Wiley, New York (1972).

[11] N.D. Birrell and P.C.W. Davies, Quantum Fields in Curved Space, Cambridge (1982).

[12] S. Chandrasekhar, The Mathematical Theory of Black Holes, Oxford (1983).

[13] M. Kasuya, Phys. Lett. 103B, 351 (1981).

[14] T. T. Wu and C. N. Yang, Nucl. Phys. B107, 365 (1976).

[15] J. M. Aguirregabiria, The Mathematica Journal, v.1, issue 2, 51 (1990).

[16] İ. Semiz, Phys. Rev. D 45, 532 (1992).

[17] R. Güven, Proc. R. Soc. A 356, 465 (1977).

[18] B. Carter and R.G McLenaghan, Phys. Rev. D 19, 1093 (1979).

[19] B. Carter and R.G McLenaghan, pp.575 of Proceedings of the Second Marcel Grossman Meeting on General Relativity, 1979, ed. R. Ruffini, North Holland (1982)

[20] R.G McLenaghan and Ph. Spindel, Phys. Rev. D 20, 409 (1979); Bull. Soc. Math. Belg. XXXI, 65 (1979).

[21] N. Kamran and R.G McLenaghan, Lett. Math. Phys. 7, 381 (1983).

[22] R. Rüdiger, J. Math. Phys. 25, 649 (1984).

[23] N. Kamran and R.G McLenaghan, J. Math. Phys. 25, 1019 (1984).

[24] B.R. Iyer and C. V. Vishveshwara, J. Math. Phys. 26, 1034 (1985). 
[25] B.R. Iyer and C. V. Vishveshwara, J. Math. Phys. 28, 1377 (1987).

[26] K. G. Suffern, E. D. Fackarell and C.M. Cosgrove, J. Math. Phys. 24, 1350 (1983).

[27] S. K. Chakrabarti, Proc. R. Soc. A 391, 27 (1984).

[28] E. G. Kalnins and W. Miller, J. Math. Phys. 33, 286 (1992).

[29] B.R. Iyer and N. Kamran, J. Math. Phys. 32, 2497 (1991).

[30] V. G. Bagrov, A. V. Shapovalov and A. A. Yevseyevich, Class. Quantum Gravity 8, 163 (1991); and references therein.

[31] G. V. Shishkin and W. D. Cabos, J. Math. Phys. 33, 916 (1992); and references therein. 